\title{
Комментарии \\ к вопросам определения стационарных движений однороторного гиростата с полостью, заполненной жидкостью большой вязкости
}

\author{
А. В. Дорошин (Самарский университет) \\ doran@inbox.ru
}

\begin{abstract}
На основе анализа научных источников дается объективная оценка новизны научных результатов, представленных в статье [1], относительно научных приоритетов по нахождению параметров стационарных режимов движения однороторных гиростатов (с полостями с жидкостью большой вязкости и без них).
\end{abstract}

\section{Введение}

Вопросы фиксации и подтверждения научных приоритетов всегда были и остаются критически важными для ученых, выполняющих свои научные исследования и квалификационные работы (диссертации) в соответствии с этическими нормами и правилами исследовательского протокола при нулевой терпимости к плагиаризмам разного рода. В этой связи целью настоящих комментариев является обозначение выявленных фактов некорректного указания в статье [1] известных ранее [2] параметров стационарных режимов движения в качестве новых научных результатов. Фактом также является отсутствие в работе [1] ссылки на работу [2].

В настоящих комментариях предметно и с необходимыми ссылками на научные источники вскрывается факт имеющегося в работе [1] плагиаризма, который, возможно, является неумышленным, однако при этом не перестает быть таковым.

Как результат, научный приоритет по параметрам стационарных режимов движения динамически симметричного однороторного гиростата фиксируется работой [2], поэтому нигде позднее и никем другим эти параметры не могут позиционироваться новым научным результатом, включая последующие публикации и результаты, выносимые на защиту в каких-либо диссертационных работах.

\section{1. Математические модели и следующие из них результаты}

\section{1. Математическая модель и параметры стационарных режимов,} приведенные в статье [2]

В статье 2002 года в журнале РАН [2] было подробно изучено свободное движение системы соосных тел как спускаемого аппарата с частичной закруткой - эта механическая модель представляет собой динамически симметричный однороторный гиростат.

В работе [2] приведены следующие динамические уравнения, которые для изучаемого случая свободного движения $\left(M_{x}=M_{y}=M_{z}=M_{\mathrm{r}, z}=M_{\delta}=0\right)$ будут иметь нулевые правые части:

$$
\begin{aligned}
& A \dot{p}-\left(A-C_{b}\right) q r+C_{r} q(r+\sigma)=0, \\
& A \dot{q}-\left(C_{b}-A\right) p r-C_{r} p(r+\sigma)=0, \\
& C_{r}(\dot{r}+\dot{\sigma})+C_{b} \dot{r}=0 . \\
& C_{r}(\dot{r}+\dot{\sigma})=0
\end{aligned}
$$

Здесь и далее нумерация формул для удобства проверок при сопоставлении работ приводится в формате (N [M]), где $\mathrm{N}$ - номер формулы в статье М. Инерционно-массовые параметры системы в интересах сопоставления обозначений с другими статьями обозначены в (5 [2]) и (6 [2]) следующим образом: $A_{b}, A_{r}, C_{b}, C_{r}$ - экваториальные и 
продольные моменты инерции динамически симметричного основного тела (индекс “ $b$ ”, обозначающий "body") и динамически симметричного тела-ротора (индекс “ $r$ ", обозначающий “rotor"); при этом $A=A_{b}+A_{r}, C=C_{b}+C_{r}$.

На основе уравнений (5 [2]) и (6 [2]) в статье [2] в разделе №4 найдены два стационарных режима (в статье [2] они не нумеровались):

$$
\begin{array}{ll}
\text { 1). } \quad p=p_{0}, \quad q=q_{0}, \quad r=r_{0}, \quad \sigma=\frac{A-C}{C_{r}} \cdot r_{0}, \\
\text { 2). } \quad p=0, \quad q=0, \quad r=r_{0}, \quad \sigma=\sigma_{0},
\end{array}
$$

где $p_{0}, q_{0}, r_{0}, \sigma_{0}$ - постоянные величины, соответствующие значениям угловых скоростей при реализации стационарных режимов движения.

\section{2. Математическая модель и параметры стационарных режимов, приведенные в статье [1]}

Приведем математическую модель, используемую в статье 2012 года, опубликованную в Вестнике СГАУ [1]. Как отмечено в статье [1], базовой моделью в работе была выбрана модель из статьи [3], в которой была построена следующая система динамических уравнений динамически симметричного однороторного гиростата с полостью с жидкостью большой вязкости (причем в [3] явно сказано об упрощенном «линеаризованном» виде модели влияния на гиростат внутренней жидкости):

$$
\begin{aligned}
& A \dot{p}-(A-C) q r+C_{r} q \sigma=\frac{\rho}{v} D \frac{s}{A}\left(C r+C_{r} \sigma\right) p, \\
& A \dot{q}+(A-C) p r-C_{r} p \sigma=\frac{\rho}{v} D \frac{s}{A}\left(C r+C_{r} \sigma\right) q, \\
& C_{r}(\dot{r}+\dot{\sigma})+C_{b} \dot{r}=-\frac{\rho}{v} D s\left(p^{2}+q^{2}\right), \\
& C_{r}(\dot{r}+\dot{\sigma})=0,
\end{aligned}
$$

где в интересах унификации также выполнено приведение обозначений величин к единому виду: $A_{b}, A_{r}, C_{b}, C_{r}$ - экваториальные и продольные моменты инерции динамически симметричного основного тела (индекс “ $b$ ”) и динамически симметричного тела-ротора (индекс “ $r$ "); при этом по-прежнему $A=A_{b}+A_{r}, C=C_{b}+C_{r}$. Параметры $D, \rho, v$ являются постоянными величинами, определяющими свойства жидкости и полости. В правых частях (14 [3]) используется переменная $s$ :

$$
s=\left[(A-C) r-C_{r} \sigma\right] A^{-1}
$$

Именно на основе уравнений (14 [3]) в работе [1] записаны условия реализации стационарного режима:

1) $p=q=0, s=u=$ const

2) $p=a=$ const, $q=b=$ const, $s=0$.

Стационарные режимы (7 [1]) позиционируются в работе [1] как новый научный результат, однако, это совершенно не так. Более того, затронутые в работе [1] вопросы анализа устойчивости режимов в первом приближении смысла не имеют, т.к. модель (14 [3]) уже записана в упрощенном (линеаризованном) виде и на самом деле не может быть объектом для «вторичной» линеаризации и линейного анализа свойств устойчивости - здесь нельзя применять анализ устойчивости по первому приближению.

Покажем, что режимы (7 [1]) полностью перезаписывают и повторяют найденные на десять лет ранее режимы (Non [2]), совершенно не отличаясь от них ни в математическом, ни в физическом смысле. 
Начнем с режима 2) из соотношений (7 [1]). Этот режим предполагает свою реализацию с постоянными величинами экваториальных угловых скоростей $p=a=$ const $=p_{0}, q=b=$ const $=q_{0}$ при нулевом значении переменной $s=0$. Указанное требование равенства нулю переменной $s$ мгновенно зануляет правые части динамических уравнений (14 [3]), которые сразу перезаписываются в однородном виде:

$$
\begin{aligned}
& A \dot{p}-(A-C) q r+C_{r} q \sigma=0, \\
& A \dot{q}+(A-C) p r-C_{r} p \sigma=0, \\
& C_{r}(\dot{r}+\dot{\sigma})+C_{b} \dot{r}=0, \\
& C_{r}(\dot{r}+\dot{\sigma})=0,
\end{aligned}
$$

что полностью редуцирует модель однороторного гиростата с полостью с жидкостью (с ее линейными свойствами влияния на тело) до уровня модели свободного однороторного гиростата, описываемого уравнениями (5 [2]), (6 [2]). Другими словами, режим, во-первых, соответствует известной модели, изученной в работе [2] и, во-вторых, полностью его повторяет, т.к. из условия $s=0$ из (Non [3]) непосредственно следует:

$$
r=r_{0}, \quad \sigma=\frac{A-C}{C_{r}} \cdot r_{0},
$$

т.е. режим в итоге представляется в виде:

$$
p=p_{0}, q=q_{0}, r=r_{0}, \quad \sigma=\frac{A-C}{C_{r}} \cdot r_{0},
$$

полностью повторяющем первый режим (Non [2]) из статьи [2]. Здесь, доводя математические модели до верхнего предела абстракции, можно сказать, что режим (Non [2]) из статьи [2] будет актуален как для системы (14 [3]), так и для любой модификации ее правой части при формальном добавлении произвольных членов, вырождающихся в ноль при условии $s=0$, например:

$$
\left\{\begin{array}{l}
A \dot{p}-(A-C) q r+C_{r} q \sigma=\frac{\rho}{v} D \frac{s}{A}\left(C r+C_{r} \sigma\right) p+\left\{n s^{2}+k s^{3}+\ldots+f \sin ^{m} s^{j}(\text { anyexpression })+\ldots\right\}, \\
A \dot{q}+(A-C) p r-C_{r} p \sigma=\frac{\rho}{v} D \frac{s}{A}\left(C r+C_{r} \sigma\right) q+\left\{l s^{2}+g s^{3}+\ldots+v \operatorname{tg}^{q} s^{e}(\text { any expression })+\ldots\right\}, \\
C_{r}(\dot{r}+\dot{\sigma})+C_{b} \dot{r}=-\frac{\rho}{v} D s\left(p^{2}+q^{2}\right)+\left\{a s^{2}+b s^{3}+\ldots+c\left[1-\exp \left(s^{v}\right)\right](\text { any expression })+\ldots\right\}, \\
C_{r}(\dot{r}+\dot{\sigma})=0+\left\{y s^{2}+o s^{3}+\ldots+w s^{h^{s}}(\text { anyexpression })+\ldots\right\},
\end{array}\right.
$$

где члены в фигурных скобках в правых частях являются принципиально гипотетическими, произвольными, но равными нулю при $s=0$. Это формально «другие» модели и другие физические основания для движения, однако, очевидно, что это простая математическая спекуляция, но даже в этом спекулятивном случае режим (Non [2]) из статьи [2] останется актуальным, т.к. он определяет базовые свойства системы однороторного гиростата (левые части уравнений).

Таким образом, режим 2), представленный соотношениями (7 [1]) из работы [1], в принципе соответствует модели свободного однороторного гиростата без жидкого наполнения [2] и полностью перезаписывает собой режим 1) из работы [2], поэтому новым результатом не является.

Режим 1) из (7 [1]) еще более тривиален и также соответствует однородному виду динамических уравнений (14 [3]) с нулевыми правыми частями, повторяющему уравнения $(5[2]),(6[2])$. 
В силу постоянных величин $\left(s=u=\left[(A-C) r_{0}-C_{r} \sigma_{0}\right] A^{-1}=\right.$ const; $r=r_{0}=$ const; $\sigma=\sigma_{0}=$ const $)$ режим 1) из (7 [1]) полностью перезаписывает второй режим (Non [2]) из работы [2]:

$$
p=0, \quad q=0, \quad r=r_{0}, \quad \sigma=\sigma_{0} .
$$

Возможно, будет уже лишним отметить, что сама попытка поиска стационарных режимов в диссипативной системе (а жидкость большой вязкости - это существенно диссипативная система) обречена на провал, т.к. физически не представляется возможным осуществление стационарного с незатухающими скоростями движения при действии жидкостного трения, стремящегося подавить к нулю любую инициацию относительной скорости, причем по экспоненциальному закону. Т.е. физического смысла в решении задачи на самом деле нет - это рассмотрение «вечного двигателя», черпающего энергию неизвестно откуда для поддержания стационарного движения системы при отсутствии внешних сил, но при наличии большого внутреннего трения (большая вязкость) от неидеальной движущейся жидкости. Справедливости ради можно отметить, что стационарное вращение в пределе станет возможным в связи с сохранением общего кинетического момента системы, но только в том случае, когда само твердое тело-носитель и все внутренние слои жидкости, отработав свое внутреннее трение на асимптотически длительном интервале движения, стали реализовывать единое угловое движение, отменив внутреннее трение по причине отсутствия относительных смещений частиц - такое предельное стационарное движение соответствует по своей сути «застыванию» внутренней жидкости и присоединению ее в виде дополнительной массы в состав единого абсолютно твердого тела, что опять же возвращает ситуацию к модели, раскрытой в [2]. Это дополнительно подтверждает в физическом плане то обстоятельство, что представленные в [1] стационарные режимы на самом деле соответствуют идеальному консервативному случаю динамики свободного динамически симметричного однороторного гиростата, рассмотренному в [2].

Другими словами, записанные стационарные режимы статьи [1] на самом деле соответствуют модели свободного однороторного гиростата без жидкого наполнения (эффекты жидкости математически исчезают при занулении правых частей динамических уравнений), повторяют и «переоткрывают» режимы, полученные на десять лет ранее в статье [2]. Никаких упоминаний результатов работы [2] в работе [1] не присутствует, равно как и не присутствует соответствующей ссылки. Указанные факты вскрывают наличие в работе [1] плагиаризма и нарушения норм исследовательского протокола, что должно быть доведено до научного сообщества сообщением в научном журнале, породившем эту ситуацию.

\section{2. Заключение}

Как показано в настоящих комментариях, вскрыт факт плагиаризма в работе [1] относительно научного результата, связанного с параметрами стационарных режимов динамически симметричного однороторного гиростата.

Научный приоритет по параметрам стационарных режимов движения динамически симметричного однороторного гиростата фиксируется работой [2], опубликованной на десять лет ранее по отношению к [1], поэтому нигде позднее и никем другим эти параметры не могут позиционироваться в качестве сколь-нибудь нового научного результата в рамках произвольной спекулятивно обобщенной математической модели, включая последующие публикации членов авторского коллектива статьи [1] (например, работы $[1,4,5])$, а также фигурировать в виде результатов, выносимых на защиту в каких-либо диссертационных работах. 


\section{Список литературы}

1. Алексеев А.В., Безгласный С.П., Красников В.С. Стабилизация стационарных движений однороторного гиростата с полостью, заполненной жидкостью большой вязкости // Вестник Самарского государственного аэрокосмического университета им. академика С.П. Королёва (национального исследовательского университета). 2012. - № 5-1 (36). - С. 13-18.

2. Асланов В. С., Дорошин А. В. Стабилизация спускаемого аппарата частичной закруткой при осуществлении неуправляемого спуска в атмосфере //Космические исследования. - 2002. - Т. 40. - №. 2. - С. 193-200.

3. Алексеев А. В. Движение спутника-гиростата, содержащего полость с жидкостью большой вязкости //Известия Самарского научного центра Российской академии наук. - 2007. - Т. 9. - №. 3.

4. Алексеев А. В., Безгласный С. П., Красников В. С. Построение стабилизирующего управления для стационарных движений гиростата с полостью с вязкой жидкостью //Известия Самарского научного центра Российской академии наук. - 2013. - Т. 15. - №. 6-3.

5. Безгласный С. П. Стабилизация стационарных движений гиростата с полостью, содержащей вязкую жидкость //Известия высших учебных заведений. Авиационная техника. - 2014. - №. 4. - С. 7-10. 